\title{
Global mental health: achievements, concerns and (unanswered) questions
}

\author{
B. Saraceno, Guest Editor* and C. Barbui, Editor
}

First published online 20 July 2016

The notion of global mental health (GMH) is widely and increasingly used in the scientific literature and in academic training; as an example, almost all important American universities have created a course of global health, which includes modules on GMH. Despite growing interest and popularity, GMH refers to concepts and contents that are quite difficult to clearly and rigorously define. Some authors like Patel \& Prince (2010) or Becker \& Kleinman (2013) have provided definitions, which capture some key concepts of GMH. However, these definitions only partially help answer a fundamental question: who is setting the GMH agenda? Four Editorials in this issue of Epidemiology and Psychiatric Sciences may hopefully contribute to address this key question (Bracken et al. 2016; Freeman, 2016; Patel, 2016; Saxena, 2016).

Indeed, addressing this issue implies raising a number of additional and somehow controversial questions (Summerfield, 2008):

- Is GMH really global or rather Western?

- Is GMH too unbalanced towards a biomedical model?

- Is GMH concerned enough with culture and local context?

- What is the real impact (if any) of GMH in lowincome countries?

- What are the consequences on the human rights of people in psychiatric institutions of the almost exclusive emphasis given by GMH on common mental disorders and on primary care level? Are people with severe mental disabilities living in institutions once again at risk to be forgotten?

- Is GMH generating resources and, if yes, where are these resources going?

The four Editorials are addressing some of these issues. Saxena (2016) offers a very pragmatic perspective from the World Health Organization (WHO):

*Address for correspondence: Professor B. Saraceno, Lisbon Institute of Global Mental Health, Nova University of Lisbon, Portugal.

(Email: benedetto.saraceno@gmail.com)
'Global mental health can quite simply be conceptualised as the highest attainable mental health for all...; following this statement Saxena provides an impressive list of WHO's recent achievements and contributions in this area, and by doing so he effectively shows that a lot has already been done but a lot still remains ahead. It follows that a too philosophical debate about what GMH is or should be is not the main WHO preoccupation. However, in the last part of his Editorial the Director of the WHO Department of Mental Health and Substance Abuse offers some important challenging considerations that should be seriously addressed: first, to which extent are global recommendations applicable to all countries in spite of the dramatic differences in terms of resources and health systems organizations? Second: to which extent can innovations in mental health care delivery be integrated into routine care on a large scale basis? Third, Saxena reminds us that GMH needs to pay a higher level of attention to the human rights of persons with mental disorders who are vulnerable to abuses within the context of mental hospitals and other similar institutions. Indeed, the Editorial does not mention any specific group of stakeholders who should be more concerned with human rights issues, but we know that it is urgent for psychiatrists to put human rights on the top of their priorities.

Patel (2016) in his visionary and quite optimistic Editorial stresses the potential rather than the actual achievements of GMH. According to Patel, the discipline of GMH has been built upon the foundations of transcultural psychiatry and global health and aims at reducing the treatment gap for mental disorders. However, GMH is more than a 'delivery science' which has been able to reframe the typology of agents delivering mental health interventions, the settings in which interventions are delivered, and the content of the interventions. Patel considers that GMH has the potential to contribute to discovery science, which aims at the identification of the aetiology of mental disorders through innovative epidemiological research, and development of new interventions, which could potentially address co-existing mental and physical 
disorders. Finally, Patel envisages the possibility of translating findings emerging from neuroscience to technologies, which can lead to scalable diagnostic tools for early detection and treatment.

With a much less optimistic vision of reality, Freeman (2016) brings the voice of low-income countries and raises a number of provocative and key questions about the cost of interventions even when they are considered cost effective and cheap by researchers: '. . . even low cost and cost-effective mental health interventions that may be possible in a research environment may just not be possible for scale up'. Freeman not only expresses reservations about the costs of interventions but goes even further by asking a radical question: is the provision of mental health interventions truly the best possible use of available resources? In other words it should not be taken for granted that scaling up treatments is the best or most cost-effective way for improving the population mental health. Freeman thinks that the problem is not just 'money' but also 'meaning', challenging this way the mental health community to reconsider the low priority given to interventions addressing social determinants rather than clinical symptoms. Social determinants are concrete factors, for example '....a depressed woman who is being regularly beaten by her husband or a depressed worker that is expected to work $15 \mathrm{~h}$ a day...'. The recent Summit jointly organised by the World Bank and WHO (Kleinman et al. 2016) made the choice of scaling up psychiatric interventions rather than addressing social determinants as the best public health choice. Freeman, and probably many other public health experts working in low-income countries, thinks that this approach might not be the most appropriate.

Finally, Bracken et al. (2016) in their Editorial offer rather substantial criticism to the foundations and epistemology of GMH. The authors consider that the movement for GMH is dominated by the western biomedical model of psychiatry, based 'on a profound, but unsubstantiated, assumption: that the mental world of human beings can be understood as simply a product of brain processes'. According to the authors, GMH pays too little attention to socio-cultural factors and by doing so it ignores the cultural dimension as if 'human emotions and behaviours can be studied independent of context'. In addition, for the vast majority of people living in low-income countries mental problems are framed in a context of poverty, and disregarding this and other contextual issues while only promoting a biomedical understanding (and treatment) of mental disorders has the potential to produce negative effects. Finally, the authors conclude that GMH is not effectively and properly hearing the voices from non-Western cultural settings and in particular those from the most vulnerable and marginalised.

These four outstanding Editorials bring more clarity about the nature, scope, content, achievements, issues and current limitations of GMH. Nevertheless, many fundamental issues and questions (some mentioned in this Presentation) remain unaddressed and will require more research and theoretical thinking. It is probably the time to keep an open dialogue among all the actors involved.

\section{Acknowledgements}

None.

\section{Financial support}

This research received no specific grant from any funding agency, commercial or not-for-profit sectors.

\section{Conflict of Interest}

None.

\section{References}

Becker AE, Kleinman A (2013). Mental health and the global agenda. New England Journal of Medicine 369, 66-73.

Bracken P, Giller J, Summerfield D (2016). Primum non nocere. The case for a critical approach to global mental health. Epidemiology and Psychiatric Sciences, this issue. doi: $10.1017 / S 2045796016000494$.

Freeman M (2016). Global mental health in low and middle income, especially African, countries. Epidemiology and Psychiatric Sciences, this issue. doi: 10.1017/S2045796016000482.

Kleinman A, Lockwood Estrin G, Usmani S, Chisholm D, Marquez P, Evans T, Saxena S (2016). Time for mental health to come out of the shadow. Lancet 387, 2274-2275.

Patel V (2016). From delivery science to discovery science: realising the full potential of global mental health. Epidemiology and Psychiatric Sciences, this issue. doi: 10.1017/ S2045796016000263.

Patel V, Prince M (2010). Global mental health: a new global health field comes of age. JAMA 3033, 1976-1977.

Saxena S (2016). Challenges and opportunities in global mental health: a perspective from WHO. Epidemiology and Psychiatric Sciences, this issue. doi: 10.1017/ S2045796016000536.

Summerfield D (2008). How scientifically valid is the knowledge base of global mental health? BMJ 336, 992-994. 